\title{
Emerging infections and global health security: the case (again) for strengthening all-hazards preparedness and response under IHR-2005
}

\author{
Jaouad Mahjour ${ }^{1}$ and Ala Alwan ${ }^{2}$
}

The World Economic Forum in Davos in 2008 highlighted the quotation by French Poet, Paul Valery, "the problem of our times is that our future is not what it used to be". Nothing could be truer today as we face outbreaks of Ebola at unprecedented levels, which threaten our social fabric globally.

What is disappointing is that we have experienced past epidemics and pandemics (SARS, H1N1, H5N1, etc.), we have anticipated and talked about the likelihood of such global calamities in the future, and we have agreed on initiatives and frameworks, such as the International Health Regulations (IHR) $(2005)^{1}$, in order to be prepared for such eventualities. But, the unfortunate fact of the matter is that we again find our global security under threat and ourselves scrambling to mount an unprecedented response to halt the spread while at the same time gaps become evident in our commitment to implementation of the agreed upon actions and capacities.

Global consensus has identified the Ebola outbreak as a worldwide security threat. The outbreak is severely damaging the economies and social life in the affected West African countries. The extraordinary exponential increase in the number of Ebola cases demands an unparalleled response that must be swift and substantial. EMRO like other

1 International health regulations (2005), 2nd ed. Geneva: World Health Organisation, 2008.
WHO regions is committed to supporting the $\mathrm{WHO}$-wide response and at the same time to advocating for and assisting response readiness for our regional Members States, especially those at high risk of importation of Ebola.

It is imperative that as we come together to mount an appropriate response to Ebola, we also focus on the weaknesses in the systems that have allowed the disease to progress from a localized outbreak to a global security threat. What is abundantly clear is that the basic principles of sound public health practice and emergency management remain our best defence against diseases like Ebola. These include: hygiene and sanitation; infection control; timely, effective and efficient surveillance; contacts tracing and isolation; comprehensive primary health care; preparedness and response; and intersectoral coordination. It is also evident that as we talk about an unprecedented response, it is only the magnitude, scale and scope of these interventions and speed of their implementation that have changed, not the types of intervention per se. These very basic approaches and actions have helped society prevail against global pandemics in the past and can help us again today, if we have the right mix of tools, speed, scale and coordination.

The IHR (2005) advocate for and emphasize strengthening of these very basic core capacities and tools at the country level, augmented with effective and efficient multisectoral coordination. If the relevant core capacities - legislation, IHR national focal point communication, surveillance (all-hazards, events-based), preparedness, response, laboratory, risk communication, human resources, points of entry - were adequately in place in any country facing such an Ebola outbreak, arguably they could alert surveillance monitors far earlier to increasing number of illnesses and deaths, many of them in clusters of close contacts.

Ebola has clearly brought to light shortcomings in public health systems, the limited resources and the deficiencies in IHR core capacities at country level. What is evident is that these limitations and deficits are ubiquitous and affect many countries; they vary only in number, scale and scope. Unless and until we all come together and address our existing weaknesses and insufficiencies, any remedial measures instigated will at best be fragmented, short-lived and symptomatic and leave the same systems exposed and perhaps even more vulnerable to the next global threat/s.

It is greatly encouraging that as a global community we are rallying to bring about a concerted response to overcome these recent threats, but we will be negligent if we do not use this opportunity to invest in core capacities, namely building/ strengthening events-based national level surveillance systems and capacities, training human resources to detect and report earlier, deploying resources strategically 
to respond better, strengthening linkages between points of entry and such systems and with neighbouring countries, identifying and mapping priority hazards and helping countries and communities to prepare better against natural hazards, communicable diseases and other manmade threats.
The current Ebola outbreak demands both immediate and sustained response and also efforts from countries to build their capacity and systems, as agreed upon in IHR, in order to be prepared for the future. This dual approach is essential to combat the current outbreaks and ensure adequate preparedness and response systems are in place for a sustainable, threat-free world. Without these systems, we are likely to be caught again and again by the emergence of other communicable disease outbreaks and other public health events that threaten global health and security. 